

EDITORIAL

\title{
IMPORTANCIA DE LA INVESTIGACIÓN DURANTE LA PANDEMIA DE COVID-19
}

\section{IMPORTANCE OF INVESTIGATION DURING THE COVID-19 PANDEMIC}

\author{
Lic. Ilya Casanova Romero, PhD1, http://orcid.org/0000-0003-1147-7413
}

1Docente e Investigadora de la Facultad de Ciencias Médicas de la Universidad Laica Eloy Alfaro de Manabí-Ecuador, Docente jubilada de la Universidad del Zulia-Venezuela. Experiencia docente y tutora en pregrado y postgrado (especialidad, maestría y doctorado). Autora de capítulos de libro y artículos en revistas arbitradas de alto impacto nacionales e internacionales.

Correo electrónico: ilya.casanova@uleam.edu.ec

2477-9172 / 2550-6692 Derechos Reservados ( 2021 Universidad Técnica de Ambato, Carrera de Enfermería. Este es un artículo de acceso abierto distribuido bajo los términos de la Licencia Creative Commons, que permite uso ilimitado, distribución y reproducción en cualquier medio, siempre que la obra original es debidamente citada.

Recibido: 10 de diciembre 2020

Aceptado: 28 de diciembre 2020

Desde la antigüedad se ha enfrentado la diatriba en cuanto a la mejor forma de asumir los procesos de investigación, cada década ha puesto en la palestra diferentes conflictos, donde en algunos casos ha privado el momento contextual; no obstante, ha sido evidente que las ideologías de quienes gestionan los procesos de investigación han tratado de prevalecer su enfoque epistemológico, el método que más se adapta a su visión o el tipo de conocimiento a generar, sin embargo, el año 2020 implicó una disrupción en las dinámicas sociales, lo cual indiscutiblemente impactó a la comunidad científica y por ende, la dinámica de la investigación.

En los inicios de la pandemia fue visible el desconocimiento sobre el impacto que tendría el SARS-CoV-2 en las distintas esferas de la sociedad, la crisis ha ido escalando y las repercusiones del COVID-19 se mantienen. Las primeras informaciones sobre este virus y las complicaciones de los pacientes fueron reservadas, lo cual generó, una respuesta tardía por parte de la comunidad científica y el único asidero era el bagaje de conocimientos, que hasta la fecha se tenían sobre otros coronavirus (1). Lo cierto, es que se aprendió y se sigue aprendiendo sobre la marcha, pero se reconoce que el trabajo en red ha sido crucial para llegar al punto de contar en la actualidad con la vacuna, algo que en condiciones de «normalidad», tomaría años. El trabajo global y conjunto, evidencia las consecuencias del impacto negativo del COVID19 en la sociedad, no obstante, se reconoce que, para la investigación en esta área, fue una oportunidad.

Por supuesto, la dinámica científica responde a unos criterios de validez y verificabilidad sobre todo en el área de la salud que ameritan tiempo y en ocasiones evitan por criterio ético la inmediatez de la información que es demandada. Esto por supuesto trajo consigo matrices de noticias falsas debido a las exigencias de información de la sociedad, que complicaron el escenario mundial, ya que, en algunos contextos, la toma de decisiones para enfrentar la crisis fue con base a estas noticias, lo cual desnudo la falta de políticas y previsiones epidemiológicas de algunos países, aun cuando, desde la academia se ha venido anunciando la posibilidad de una situación pandémica como la actual (2).

Para adaptarse a esta situación, las revistas de alto impacto decidieron que los artículos de COVID-19 serían publicados en acceso abierto (Open Access), beneficiando a la comunidad académica, sobre todo a los países latinoamericanos que, debido a los altos costos son poco utilizados, esta decisión provocó que revistas independientemente de su área del saber, se sumaran e inclusive disminuyeran los tiempos en el arbitraje de los artículos relacionados a la pandemia originada por el SARS-CoV-2, permitiendo que la difusión de los resultados proporcione redes generadoras de conocimiento que hasta hoy día se mantienen, en un mundo que entendió la nueva dinámica global, donde el trabajo interdisciplinario es indispensable, ya que se esperan nuevos eventos epidemiológicos.

Ahora bien, es innegable el protagonismo tanto de las Universidades e Institutos como del equipo de salud en esta crisis, se ha destacado, por ejemplo, el repunte en los resultados de los programas de investigación a quienes se les suministró del financiamiento suficiente e inmediato, lo cual permitió generar conocimientos sobre temas orientados a la descripción del nuevo coronavirus, reporte de estudios clínicos y de tratamientos propuestos para enfrentar el COVID-19 $(3,4)$. Otro de los aspectos positivos que emerge es la responsabilidad de los propios investigadores en esta una nueva era, de la necesidad de asumir su identidad digital en las redes sociales académicas y resaltar que no es suficiente realizar proyectos con pares nacionales o muy a lo interno de las instituciones donde están adscritos y publicar algunos artículos o presentar una conferencia en un congreso internacional (5).

Algunos investigadores por supuesto quedaron como paralizados en la pandemia y dejaron en pausa sus proyectos de investigación, no obstante, se evidenció que luego de salir de la conmoción, la mayoría de los grupos de investigación entendieron, que era el momento de buscar nuevas rutas operativas, que les permitiera llevar a cabo los procesos investigativos y esto implica madurez en la ciencia, proyectándose así a un cambio y una comprensión de esa lógica evolutiva darwiniana de la investigación. Se hizo evidente que no hay recetas investigativas, que el conocimiento científico no tiene fecha de vencimiento, que es importante realizar revisiones diacrónicas en la temática seleccionada, que permitan identificar los vacíos en el conocimiento, sobre todo, seleccionar el método coherente con el enfoque epistemológico asumido. 
Otro de los aspectos que se está valorando en la comunidad científica son las buenas prácticas en investigación, dentro de las que destaca el reportar resultados aun cuando estos sean contrarios a la hipótesis de la investigación. Reportar estos resultados reducen los costos financieros para otras investigaciones y permiten avanzar en conjunto en los programas de investigación relacionados; la rivalidad de los grupos puede ser ya un planteamiento obsoleto, al inclinarse la balanza hacia la resolución conjunta de problemas, que en el caso de las Ciencias de la Salud va más allá de la ausencia de enfermedad, sino que está orientado a preservar la salud y el bienestar de la población.

La pandemia aceleró el asumir nuevos retos, se van dejando atrás algunos mitos sobre cómo resolver problemas, se entiende la diversidad en la búsqueda del conocimiento, sin embargo, donde no hay concesiones por parte de la comunidad científica, es en las dinámicas y procesos que preserven la credibilidad de la ciencia. Este tiempo fortaleció los movimientos mundiales para reconocer que se deben realizar acciones para evitar las malas prácticas en investigación (6), estas malas prácticas que tal como lo plantea Ordorika fueron desviaciones producto en algunos casos, de privilegiar como indicador para el avance y permanencia en el mundo académico, solamente la publicación de artículos en revistas indexadas y el conteo de citas en los distintos índices (7).

Es el momento de las buenas prácticas en investigación, para enfrentar esta etapa disruptiva con ética, evitar los protagonismos que no favorece el avance de la ciencia fortalecer las políticas antiplagio, salir del confort de permanecer solo en una disciplina, ya que al quitarse las gríngolas disciplinares se tendrá una visión integral, para acercase por ejemplo, al logro de los Objetivos de Desarrollo Sostenible, donde el tercer objetivo orienta a la salud y el bienestar como un hecho donde se rompen esas fronteras disciplinares (8), que aíslan y conducen a una endogamia académica, que muy poco beneficia la resolución de problemas complejos en el área de la salud.

Mucho de lo descrito con anterioridad, debe ser asumido desde los campus universitarios, la educación promueve la transformación de la sociedad, es importante asumir que se debe producir conocimiento científicamente validado, compartirlo no solo con la comunidad científica sino también, luego de su transposición didáctica, con los estudiantes y la sociedad. La experiencia de esta era, ha demostrado que en algunos casos y sobre todo en el área de la salud existe una vertiginosa obsolescencia del conocimiento.

La formación de investigadores no debe ser entendida como la acumulación de horas en el aula, aprender a hacer investigación debe ser en la acción, desde la vivencia de ser investigadores, sobre este tema Padrón resalta, que parte de la responsabilidad está ubicada en la gestión de los currículos donde se ha prevalecido el carácter profesionalizante de la investigación, la cual es engullida por éste y queda atrapada en el aula por las clases de metodología y los seminarios de investigación o de proyecto, dirigidos a menudo por profesores que rara vez investigan (9). Para ser partícipes en el colectivo de investigación post-pandemia "se debe contar con una disposición positiva para generar conocimiento, lo que responde a un conjunto de creencias, actitudes e informaciones, valores, habilidades, competencias que debe desarrollar el sujeto y el colectivo" (10).

El pasado, presente y futuro de la investigación, estará marcada durante muchos años por la Pandemia del COVID19 , es un antes y un después en todas las áreas del saber, ya que la afectación no está circunscrita solo a la salud, aceptar el reto de ver más allá, queda vigente lo pronunciado por Winston Churchill en su tiempo "Los imperios del futuro son los imperios de la mente".

\section{REFERENCIAS}

1 Bravo-García E, Magis-Rodríguez C. La respuesta mundial a la epidemia del COVID-19: los primeros tres meses. Boletín sobre COVID-19 Salud Pública y Epidemiología. Departamento de Salud Pública de la Facultad de Medicina de la UNAM [revista en internet]. 2020;1(1):1-8. Disponible en: http://dsp.facmed.unam.mx/wp-content/uploads/2013/12/COVID-19-No.103-La-respuesta-mundial-a-la-epidemia-del-COVID-19-los-primeros-tres-meses.pdf

2 Catalán-Matamoros, Daniel. La comunicación sobre la pandemia del COVID-19 en la era digital: manipulación informativa Fake News y redes sociales. RECS.2020;(Suplemento 1):S5-S8. Disponible en: https://doi.org/10.20318/recs.2020.5531

3 Marchiori Buss, Paulo y Tobar, Sebastián. La COVID-19 y las oportunidades de cooperación internacional en salud. Cad. Saúde Pública 2020;36(4):e00066920. Disponible en: https://doi.org/10.1590/0102-311X00066920

4 Ortiz-Núñez Roelvis. Análisis métrico de la producción científica sobre COVID-19 en SCOPUS. Rev. cuba. inf. cienc. salud. 2020;1(3):e1587.

Disponible en: http://scielo.sld.cu/scielo.php?script=sci arttext\&pid=S2307-21132020000300002\&lng=es

5 Artigas, Wileidys y Casanova, llya. Influencia de las redes sociales académicas en la construcción de la identidad digital latinoamericana. Anales de Documentación. 2020;23(2):1-13. Disponible en: http://dx.doi.org/10.6018/analesdoc.397551

6 Artigas, Wileidys y Casanova, llya. Malas prácticas en investigación: una discusión necesaria sobre integridad académica. Integridad Académica. 2020;4(8):9-11.

Disponible en: https://issuu.com/integridadacademica/docs/revista ia - ene -jun 2020 - digital

7 Ordorika, Imanol. Las trampas de las publicaciones académicas. Revista Española de Pedagogía. $2018 ; 76$ (271):463-480. Disponible en: https://doi.org/10.22550/REP-3-2018-04

8 Programa de las Naciones Unidas para el Desarrollo (PNUD). (S.F). Objetivo 3: Salud y bienestar. Disponible en: https://www.undp.org/content/undp/es/home/sustainable-development-goals/goal-3-good-health-and-well-being.html

9 Padrón, José. Obstáculos para una investigación social orientada al desarrollo. Seminario Nacional de Ciencias Sociales en la Universidad de Carabobo. 2009, Disponible en: http://www.ing.uc.edu.ve/dhcs/

10 Casanova Romero, llya, Paredes Chacín, Ítala, Naranjo Toro, Miguel y Padrón Guillén, José. Capítulo I. Retos en la Formación de Investigadores en Contextos Universitarios. En Formación de Investigadores en el Contexto Universitario. Editorial UTN. Primera Edición. ISBN 978-9942-784-69-8. Ecuador. 2020. Disponible en:

https://issuu.com/utnuniversity/docs/formaci $\mathrm{n}$ de investigadores en el contexto univers 